\title{
Philosophiques
}

\section{La critique des anthropologies et le Discours sur l'inégalité de J.J. Rousseau}

\section{Vinh-De Nguyen}

Volume 13, numéro 2, automne 1986

URI : https://id.erudit.org/iderudit/203319ar

DOI : https://doi.org/10.7202/203319ar

Aller au sommaire du numéro

Éditeur(s)

Société de philosophie du Québec

ISSN

0316-2923 (imprimé)

1492-1391 (numérique)

Découvrir la revue

Citer cet article

Nguyen, V.-D. (1986). La critique des anthropologies et le Discours sur l'inégalité de J.J. Rousseau. Philosophiques, 13(2), 253-266.

https://doi.org/10.7202/203319ar
Résumé de l'article

Cette étude se veut le commentaire de la proposition qui ouvre la Préface du Discours sur l'Inégalité de Rousseau : « La plus utile et la moins avancée de toutes les connaissances humaines me paraît être celle de l'homme. »

Elle tente d'expliquer, du point de vue de Rousseau, le retard de la connaissance anthropologique par rapport aux autres branches du savoir, en analysant la critique faite par ce dernier à l'endroit des anthropologies marquantes de l'époque : celles du Christianisme, de Thomas Hobbes et de Buffon.

L'analyse laisse entrevoir que la Bonté naturelle et la Liberté constituent le double principe fondamental de la conception rousseauiste de l'homme, montrant du même coup que le Discours sur l'Inégalité, en tant que discours anthropologique, est une tentative de démonstration de ce principe.
Tous droits réservés @ Société de philosophie du Québec, 1986
Ce document est protégé par la loi sur le droit d'auteur. L'utilisation des services d'Érudit (y compris la reproduction) est assujettie à sa politique d'utilisation que vous pouvez consulter en ligne.

https://apropos.erudit.org/fr/usagers/politique-dutilisation/ 


\title{
LA CRITIQUE DES ANTHROPOLOGIES ET LE DISCOURS SUR L'INÉGALITÉ DE J.J. ROUSSEAU
}

\author{
par Vinh-De Nguyen
}

RESUME Cette étude se veut le commentaire de la proposition qui ouvre la Préface du Discours sur l'Inégalité de Rousseau : "La plus utile et la moins avancée de toutes les connaissances humaines me paraît être celle de l'homme.»

Elle tente d'expliquer, du point de vue de Rousseau, le retard de la connaissance anthropologique par rapport aux autres branches du savoir, en analysant la critique faite par ce dernier à l'endroit des anthropologies marquantes de l'époque : celles du Christianisme, de Thomas Hobbes et de Buffon.

L'analyse laisse entrevoir que la Bonté naturelle et la Liberté constituent le double principe fondamental de la conception rousseauiste de l'homme, montrant du même coup que le Discours sur l'Inégalité, en tant que discours anthropologique, est une tentative de démonstration de ce principe.

ABSTRACT. This article puts forward a commentary on the opening statement of the preface of Rousseau's Discourse on Inequality: "The most useful and the least advanced of all human knowledge seems to me to be that of man.»

It attempts to account for the blame laid by Rousseau on the conceptions of man of his time, by analyzing his criticisms which are meant for the prominent anthropologies of Christianity, Hobbes and Buffon.

The analysis reaches the following conclusion: The Discourse on Inequality is an attempt to vindicate what constitute the fundamental principles of Rousseau's anthropology, viz. natural Goodness and Freedom.

Après avoir étudié l'homme toute ma vie, j'avais cru connaître les hommes; je m'étais trompé. Je ne parvins jamais à en connaître un seul. ${ }^{1}$

1. Rousseau, Euvres complètes, Pléiade, I, p. 782. 
Bien avant de connaître cette déception à l'égard des hommes, - déception dont il fait part à ses lecteurs dans les Dialogues -, Rousseau a déjà éprouvé le même sentiment à l'égard de toute l'anthropologie de l'époque : «La plus utile et la moins avancée de toutes les connaissances humaines me paraît être celle de l'homme», écrit-il dans la préface du Discours sur l'Inégalité ${ }^{2}$.

Comment s'explique ce retard de l'anthropologie par rapport aux autres branches du savoir? Quels reproches adresse Rousseau aux conceptions anthropologiques de l'époque?

Notre étude ne prétend pas retrouver toutes les conceptions de l'homme auxquelles s'est opposé l'auteur de l'Émile. Elle tente de suivre ce dernier dans sa critique des anthropologies qui ont le plus longuement retenu son attention. Il s'agit des anthropologies religieuse du Christianisme, philosophique de Hobbes et «scientifique» de Buffon.

\section{CRITIQUE DE L'ANTHROPOLOGIE CHRÉTIENNE}

Qu'elle soit calvinienne ou augustinienne ${ }^{3}$, l'anthropologie chrétienne affirme que le mal sous toutes ses formes provient de la nature humaine et que cette nature est foncièrement mauvaise du fait du péché originel.

Dans ses diverses œuvres, à commencer par le Mémoire à M. de Mably jusqu'aux Lettres écrites de la Montagne, en passant par la Lettre à Beaumont, Rousseau n'a cessé de combattre cette doctrine du péché originel.

Il commence par déclarer que celle-ci manque de tout fondement scripturaire. Il n'est pas du tout certain que ce dogme soit l'enseignement de l'Évangile: «Il s'en faut bien, selon moi, que cette doctrine du péché originel, sujette à des difficultés si terribles, ne soit contenue dans l'Écriture ni si clairement ni si

2. Euvres complètes, Pléiade, III, p. 122 ; «Ce qui nous est précisément le moins connu est ce qu'il nous importe le plus de connaître, savoir l'homme.» (O.C., Pléiade, IV, p. 1092).

3. Pour un exposé des conceptions de Calvin, voir Jean BoIsSET, Calvin et la souveraineté de Dieu, Paris, Seghers, 1964, p. 139 et suiv.; sur l'anthropologie augustinienne, on peut lire Henri RONDET, «L'anthropologie religieuse de saint Augustin», dans Essais sur la théologie de la grâce, Paris, Beauchesne, 1964, p. 14 et suiv. 
durement qu'il a plu au rhéteur Augustin et à nos théologiens de la bâtir ${ }^{4}$.

Ces théologiens, ajoute Rousseau, n'ont pas vu que l'idée de péché originel contredit l'affirmation d'un Dieu bon et juste. Comment, en effet, concilier ces attributs divins avec la thèse selon laquelle Dieu crée "tant d'âmes innocentes et pures, tout exprès pour les joindre à des corps coupables, pour leur y faire contracter la corruption morale et pour les condamner toutes à l'enfer sans autre crime que cette union qui est son ouvrage. ${ }^{5}$

Il y a plus grave. Cette thèse souffre elle-même d'une contradiction interne. En effet, d'une part le péché originel est effacé par le sacrement du baptême, d'autre part le mal que l'on voit lui est imputé; comment le péché originel peut-il être à l'origine du mal alors que l'homme baptisé en est déjà délivré ? : «Selon cette même doctrine, nous avons tous dans notre enfance recouvré l'innocence primitive; nous sommes tous sortis du baptême aussi sains de cœur qu'Adam sortit de la main de Dieu. Nous avons, direz-vous, contracté de nouvelles souillures; mais puisque nous avons commencé par en être délivrés, comment les avons-nous derechef contractées ? ${ }^{6}$

Même si on ne regarde pas à ces contradictions, on sera quand même forcé d'admettre que la thèse du péché originel n'est pas à l'abri de toute objection. Élaborée pour rendre compte du mal, elle laisse inexpliquée la racine même du mal. En rendant le péché d'Adam responsable de la corruption de la nature humaine avec toutes ses conséquences, elle admet que ce péché est dû à une autre cause. Mais, demande Rousseau, pourquoi n'explique-t-on pas tout le mal par cette même cause ? Si on l'avait fait, on aurait pu faire l'économie de cette doctrine du péché originel: "Le péché originel explique tout excepté son principe et c'est ce principe qu'il s'agit d'expliquer. ${ }^{7}$ Cette critique laisse entrevoir que pour Rousseau, le mal est l'œuvre de la liberté humaine, car comment expliquer la faute du premier homme dans son état originel autrement que par un libre choix?

4. O.C., Pléiade, IV, p. 937.

5. Id., p. 938.

6. Ibid.

7. O.C., Pléiade, IV, p. 939. 
Rousseau ne s'est pas contenté de montrer ce qu'il y a de fragile et d'incohérent dans la doctrine du péché originel. Dans un fragment inédit, on le voit pousser plus loin sa critique en donnant du péché originel une autre interprétation: de ce péché il résulterait plutôt un ennoblissement pour l'homme et non une déchéance. "Au lieu d'avilir sa nature, écrit Rousseau, il semble selon moi que le péché d'Adam l'ait ennoblie en développant son esprit et le rendant capable de raison ${ }^{8}$."

Cette interprétation met en évidence ce à quoi tend toute la critique de Rousseau : la nature originelle de l'homme n'est point mauvaise et n'est donc en rien responsable du mal que les hommes subissent et se font entre eux.

Mais cette thèse de la bonté naturelle de l'homme n'est pas assurée par le seul fait de la fragilité de la doctrine du péché originel. Peut-elle se maintenir face à l'anthropologie de Hobbes?

\section{CRITIQUE DE LA CONCEPTION HOBBESIENNE}

\section{DE L'HOMME}

On le sait, pour le philosophe de Malmesbury, l'homme est méchant et égoïste. «Il est également vrai, écrit Hobbes dans son Épittre dédicatoire du $D e$ Cive, et qu'un homme est un dieu à un autre homme, et qu'un homme est aussi un loup à un autre homme ${ }^{9}$.» Mais cette méchanceté n'est pas aussi naturelle que l'égoïsme puisque Hobbes considère que «les affections de l'âme qui viennent de la nature animale, ne sont point mauvaises en elles-mêmes ${ }^{10}$.» La méchanceté se définit en fonction des devoirs que l'homme a à faire et par l'absence de la raison qui les lui fait connaître: «La méchanceté n'est autre chose que le défaut de raison en un âge auquel elle a accoutumé de venir aux hommes, par un instinct de la nature, qui doit être alors cultivée par la discipline, et qui se trouve déjà assez instruite par l'expérience des dangers et des infortunes passées ${ }^{11}$.» Dans cette perspective,

8. Rousseau cité par Bernard GAGNEBIN, «J.J. Rousseau : sur le péché d'Adam et le salut universel. Fragment inédit.» in Dix-buitième siècle, $\mathrm{n}^{\circ} 3,1971$, p. 42.

9. Hoвbes, Le Citoyen, trad. franç. par Samuel SoRBIERE, Introd. et notes par Simone GoYARD-FABRE, Paris, Flammarion, 1982, p. 83.

10. Id., p. 73.

11. HobBes, op. cit., p. 73. 
l'enfant, étant privé de l'usage de la raison, ne peut être jugé méchant quoique ses actions puissent aller contre ses devoirs. Ces mêmes actions, venant d'un adulte, dénonceraient la méchanceté de leur auteur: il agit comme s'il n'avait pas de raison; il est une espèce d' "enfant robuste», il est "l'homme qui a l'âme d'un enfant ${ }^{12}$.»

Ainsi, pour Hobbes, la méchanceté n'est pas originelle. Ce qui est originel, c'est un ensemble de dispositions qui facilitent son apparition: "Si ce n'est... que l'on veuille dire, que la nature a produit les hommes méchants, parce qu'elle ne leur a pas donné en les mettant au monde les disciplines, ni l'usage de la raison, il faut avouer qu'ils peuvent avoir reçu d'elle le désir, la crainte, la colère, et les autres passions de l'âme sensitive, sans qu'il faille l'accuser d'être cause de leur méchanceté ${ }^{13}$.»

Parmi ces dispositions, l'égoïsme est la plus fondamentale. L'homme naturel de Hobbes est foncièrement égoïste. Ce qu'il recherche d'abord et avant tout, c'est son bien-être; ce qu'il a en vue dans ses actions, c'est toujours son profit ou sa «fierté». Hobbes a tant mis l'accent sur ce trait de la nature humaine qu'on l'a appelé le philosophe de l'égoïsme ${ }^{14}$.

L'auteur du Léviathan n'est certainement pas le premier ni le seul à professer cette thèse de l'égoïsme naturel. Il nous faut cependant reconnaître que chez Hobbes, contrairement en cela aux autres penseurs, cette thèse a un caractère radical: l'homme n'a en lui-même aucun instinct qui le porte spontanément vers ses semblables. La thèse hobbésienne de l'égoïsme exclut absolument celle de la sociabilité naturelle. À ceux qui invoquent le fait de la société et de ses institutions qui paraît contredire une telle affirmation, Hobbes répondra que, plus on étudie les "affaires humaines», plus on est convaincu que "toutes les sociétés sont bâties sur le fondement de la gloire et des commodités de la vie; et qu'ainsi elles sont contractées par l'amour-propre plutôt que par une forte inclination que nous ayons pour nos

12. Id.

13. Id.

14. Sur ce point, voir Jim MacADAM, «Rousseau's criticism of Hobbesian Egoism» in Etudes Rousseau Trent, éditées par J. MacAdam, Michel Neumann et Guy Lafrance, Ottawa, Éd. de l'Université d'Ottawa, 1980, p. 121. 
semblables ${ }^{15}$.» La proposition aristotélicienne «l'homme est un animal politique», longtemps considérée comme un axiome intangible, n'est aux yeux de Hobbes qu'une grossière erreur due à « une trop légère contemplation de la nature humaine ${ }^{16}$.»

A cette anthropologie de nature philosophique, Rousseau a fait plusieurs critiques relevant de différents ordres.

Tout d'abord, une critique d'ordre méthodologique: Hobbes n'a pas vu que l'on ne comprend les faits humains qu'en les étudiant selon la perspective historique. L'esprit de cette méthode - applicable à toute recherche dans les sciences sociales - est affirmé dans le texte suivant du Second Discours: "C'est dans [la] lente succession des choses qu'il [le lecteur] verra la solution d'une infinité de problèmes de morale et de politique que les philosophes ne peuvent résoudre. Il sentira que le Genre-humain d'un âge n'étant pas le Genre-humain d'un autre âge, la raison pourquoi Diogène ne trouvait point d'homme, c'est qu'il cherchait parmi ses contemporains l'homme d'un temps qui n'était plus ${ }^{17}$.»

L'ignorance de cette dimension historique des choses humaines liée à la "méthode analytique» de Hobbes, a facilité la confusion, chez ce dernier, de la psychologie de l'homme naturel et de celle de l'homme social: «Un philosophe superficiel observe des âmes cent fois repétries et fermentées dans le levain de la société et croit avoir observé l'homme. Mais pour le bien connaître, il faut savoir démêler la gradation naturelle de ces sentiments et ce n'est point chez les habitants d'une grande ville qu'il faut chercher le premier trait de la nature dans l'empreinte du cour humain ${ }^{18}$.»

De cette erreur d'ordre méthodologique découle l'erreur psychologique, qui consiste à attribuer à l'homme naturel les sentiments, les passions et les comportements propres à l'homme social. Hobbes, dit Rousseau, a «fait entrer mal à propos dans le soin de la conservation de l'homme sauvage, le besoin de satisfaire une multitude de passions qui sont l'ouvrage de la société, et qui ont rendu les lois nécessaires ${ }^{19}$.» De là vient

15. Hоввеs, Le citoyen, p. 92.

16. Ibid., p. 90.

17. CEuvres complètes, Pléiade, III, p. 192.

18. Ibid., p. 612.

19. Ibid., p. 153 
l'accent mis sur la volonté de nuire et la méchanceté des hommes naturels.

Rousseau a renforcé sa critique en montrant que les éléments par lesquels Hobbes définit la méchanceté, à savoir la force et l'absence de raison, ne font nullement naître des comportements méchants. En effet, dire que la méchanceté « n'est autre chose que le défaut de raison», c'est implicitement reconnaître que le développement de la raison est la condition de la moralité, que la connaissance des règles et des vices empêche l'homme de mal faire : ce n'est point là une thèse qui puisse tenir devant l'auteur du Discours sur les sciences et les arts, pour qui le luxe, la dissolution des mœurs et l'esclavage sont «de tout temps le châtiment des efforts orgueilleux que nous avons faits pour sortir de l'heureuse ignorance où la sagesse éternelle nous avait placés».

De plus, la méchanceté ne peut être le fait d'un homme fort, car «celui qui pourrait tout ne ferait jamais de mal ${ }^{20}$.» Dieu n'est-il pas à la fois l'Être tout-puissant et infiniment bon ? ${ }^{21}$

La psychologie de Hobbes pèche encore par un autre vice: Hobbes ignore la réalité et le rôle de la pitié. La thèse d'un égoïsme radical et absolu n'a sans doute pas permis à Hobbes de reconnaître à l'homme naturel nulle conscience ouverte sur autrui. Rousseau déclare une telle vue inadmissible puisqu'elle ne s'accorde pas avec l'expérience, qui montre les multiples manifestations de la pitié :

Sans parler de la tendresse des mères pour leurs petits, et des périls qu'elles bravent pour les en garantir, on observe tous les jours la répugnance qu'ont les chevaux à fouler aux pieds un corps vivant; un animal ne passe point sans inquiétude auprès d'un animal mort de son espèce : il y en a même qui leur donnent une sorte de sépulture; et les tristes mugissements du bétail entrant dans une boucherie annoncent l'impression qu'il reçoit de l'horrible spectacle qui le frappe. ${ }^{22}$

Rousseau ne craint pas de présenter ces comportements animaux comme signes de la pitié parce que celle-ci est pour lui

20. CEuvres complètes, Pléiade, IV, p. 288.

21. «De tous les attributs de la divinité toute-puissante, la bonté est celui sans lequel on la peut le moins concevoir." (id).

22. O.C., III, p. 154. Voir remarques de Roger D. MASTERS sur ce texte dans son ouvrage, The Political Philosophy of Rousseau, Princeton University Press, New Jersey, 1968, p. 140. 
une qualité fondamentale de l'être sensible ${ }^{23}$. Il admet avec Hobbes que l'amour de soi est une tendance fondamentale de l'être humain, mais, à l'encontre de ce dernier, précise que cet amour ne s'exerce pas de façon brutale et aveugle, tempéré qu'il est par une « répugnance innée à voir souffrir son semblable». Ce qui, aux yeux de Rousseau, correspond à la description hobbésienne de l'égoösme, c'est plutôt l'amour-propre, «sentiment factice et né dans la société qui porte chaque individu à faire plus de cas de soi que de tout autre, qui inspire aux hommes tous les maux qu'ils se font mutuellement, et qui est la véritable source de l'honneur ${ }^{24}$.»

Dans la supposition d'un état anté-juridique, anomique que se donne Hobbes, la logique de la thèse de l'égoïsme mène immanquablement à l'affirmation de l'état de guerre comme étant naturel aux hommes, et par conséquent à celle de la paix comme bien fondamental. Ce que recherchent les hommes, par nature a-sociaux, en s'associant entre eux, c'est d'abord et avant tout, la sécurité. Celle-ci n'est possible que quand les hommes conviennent entre eux « de céder de leurs prétentions et de leurs droits sur toutes choses ${ }^{25}$ au profit du Souverain, qui ne doit satisfaire qu'à une seule exigence : réussir dans son entreprise. Il faut que, par son action, «chacun soit tellement à couvert de la violence des autres, qu'il puisse en repos et sans être en crainte perpétuelle, lorsqu'il ne fera tort à personne ${ }^{26}$.» Ce n'est qu'à cette condition que le pacte générateur de la société civile garde sa signification.

Aux yeux de Rousseau, cette conception hobbésienne sur la fin du Pouvoir politique implique une thèse anthropologique inacceptable: l'homme désire moins la liberté que la sécurité puisqu'il se soumettrait volontiers au Souverain si celui-ci le protégeait. Comparant cet état de sécurité et de soumission des hommes civils à celui des Grecs enfermés dans l'antre du Cyclope (cette comparaison est injuste puisque la sécurité des prisonniers du Cyclope n'est qu'apparente) Rousseau reproche à Hobbes de n'avoir pas vu que la renonciation à la liberté est en même temps

23. Voir O.C., III, p. 126.

24. Ibid., p. 219.

25. HoBвEs, Le citoyen, p. 74.

26. Ibid., p. 150. 
une renonciation à la nature d'homme, sans qu'il y ait nulle compensation appréciable ${ }^{27}$.

En résumé, face à la vision hobbésienne de l'homme, Rousseau oppose une négation: l'homme n'est pas naturellement méchant, et une affirmation: l'homme n'est homme que dans l'indépendance.

Ces assertions demeurent-elles valables face à une approche «scientifique» de la nature humaine?

\section{CRITIQUE DE LA CONCEPTION NATURALISTE DE BUFFON}

On le sait, Buffon est le premier à tenter une telle approche dans l'étude de l'homme. L'œuvre d'histoire naturelle de ce naturaliste marque un nouveau tournant dans l'histoire de la pensée anthropologique. Concluant son chapitre sur l'«histoire naturelle dans la culture des Lumières», Georges GUSDORF énonce ce jugement: "Depuis le siècle des Lumières, l'histoire naturelle est pour l'homme un miroir, sur lequel il se penche pour $\mathrm{y}$ retrouver, per speculum in aenigmate, les étymologies de la condition humaine. L'homme qui, naguère, découvrit dans la seule Écriture sainte les secrets de sa destinée, la recherche désormais dans l'étude du grand livre de la nature. L'anthropologie cesse d'être un chapitre de la théologie pour devenir une science de la nature ${ }^{28}$.» Il faudrait ajouter qu'avec Buffon, l'anthropologie a cessé d'être un chapitre de la pure philosophie. L'auteur de l'Histoire naturelle s'est toujours efforcé de se maintenir sur le «terrain scientifique» ${ }^{29}$.

Que cette volonté de faire œuvre scientifique ait conquis le respect, la confiance de Rousseau, est attesté par les témoignages

27. «Renoncer à sa liberté, c'est renoncer à sa qualité d'homme, aux droits de l'humanité, même à ses devoirs. Il n'y a nul dédommagement possible pour quiconque renonce à tout. " (O.C., III, p. 356.)

28. Georges GuSDORF, Dieu, la nature et l'homme au siècle des Lumières, Paris, Payot, 1972, p. 269.

29. Sur ce qui définit la scientificité de l'entreprise anthropologique de Buffon, voir Jacques ROGER, Les sciences de la vie dans la pensée française du XVIII siècle, Paris, A. Colin, 1971, p. 538; voir aussi Michèle DUCHET, «L'anthropologie de Buffon» in BUFFON, De l'bomme, Paris, F. Maspéro, 1971, p. 9-10. 
dans son ouvre et dans sa vie. C'est en pensant à Buffon qu'il écrit ces lignes: "Dès mon premier pas, je mappuie avec confiance sur une de ces autorités respectables pour les philosophes, parce qu'elles viennent d'une raison solide et sublime qu'eux seuls savent trouver et sentir ${ }^{30}$.»

Le traité De l'homme de Buffon affirme l'éminente dignité de l'homme par rapport à tous les animaux. Cette affirmation repose sur des faits aisément observés par tout le monde. Il s'agit en premier lieu de la réflexion c'est-à-dire le pouvoir qu'a l'homme, même le plus stupide, de se donner «un projet raisonné, un ordre d'action et une suite de moyens» qui lui permettent de contraindre les bêtes à lui obéir. La subordination de celles-ci n'est nullement due à la force ou à l'adresse de l'homme, puisqu'on n'a jamais vu les animaux les plus forts, les plus adroits commander aux autres. Le langage est le deuxième fait qui distingue l'homme de l'animal. Buffon considère que le langage est essentiellement dû au pouvoir de réflexion: l'homme parle parce qu'il est un être pensant. La preuve, c'est que le singe ne parle pas, quoiqu'il ait la langue "aussi parfaite que celle de l'homme». Cette absence de la pensée explique encore l'incapacité des animaux de créer et de faire des progrès. Inversement, c'est parce qu'il est doué de pensée que l'homme est perfectible ${ }^{31}$.

Ces trois faits : réflexion, langage et perfectibilité démontrent incontestablement la spiritualité de l'âme humaine. Admettre cette conclusion, c'est en même temps admettre qu'il y a solution de continuité dans la chaîne des êtres et c'est retrouver, par un détour objectif, la conception traditionnelle pour laquelle « l'homme est un être raisonnable, l'animal est un être sans raison ${ }^{32}$.»

L'approche «scientifique» ne permet pas seulement à Buffon de souligner la différence spécifique de l'homme par rapport aux

30. O.C., III, p. 195; voir aussi Otis Fellows, «Buffon and Rousseau: Aspects of a Relationship" in Publication of the Modern Language Association of America, vol. LXXXV, 1960, June n. 3, p. 184-196; Correspondance complète, édition Ralph A. LeIGH, lettre $n^{\circ} 3326$, le ministre J.H. Meister au pasteur J.H. Meister le 6 juin 1764, vol. XX, p. 151; lettre $n^{\circ} 3602$ à Du Peyrou le 4 novembre 1764, vol. XXII, p. 5 et vol. XXXVIII, Notes de Henri Nadault de Buffon, p. 320 ; O.C., IV, p. 382 , note**.

31. Voir BUFFon, ouv. cité, p. 45.

32. Buffon, ouv. cité, p. 47. 
animaux, mais encore de mettre en évidence la différence de l'homme par rapport à lui-même dans le temps. Grâce à sa raison, l'homme se perfectionne: avant il était sauvage, "sujet à toutes les lois et même aux excès de la nature» ${ }^{33}$, aujourd'hui il est civilisé et est devenu le conquérant et le maître du monde. L'optimisme rationaliste dont Daniel MORNET fait la caractéristique des "philosophes» du $18^{\mathrm{e}}$ siècle français, trouve son fondement scientifique chez Buffon.

Ce progrès est aussi un progrès moral. Pour Buffon, ce qui différencie les hommes policés des sauvages, ce n'est pas seulement les réalisations culturelles prodigieuses, mais c'est encore la moralité. S'il en est ainsi, civiliser les peuples sauvages serait un devoir pour les peuples policés. «Rien ne fait plus d'honneur à la religion, écrit Buffon en parlant du succès des missionnaires auprès des «sauvages» du Paraguay, que d'avoir civilisé ces nations et jeté les fondements d'un empire sans autres armes que celles de la vertu ${ }^{34}$.» Commentant ce texte et d'autres qui expriment l'européocentrisme du naturaliste, Michèle DUCHET a raison d'écrire: «... l'anthropologie de Buffon donnait un fondement philosophique à un nouveau modèle de colonisation ${ }^{35}$.»

Qu'il y ait une grande influence de Buffon sur la pensée anthropologique de Rousseau est chose certaine. Dans une étude publiée en 1909 sur les sources du Second Discours, Jean MOREL a montré que c'est chez Buffon que Rousseau puise son information scientifique. C'est le naturaliste qui lui a suggéré l'idée de perfectibilité, la distinction du physique et du moral dans l'amour, l'idée de l'expérience du croisement dont il parle de façon allusive dans la note $\mathrm{X}$ de son Discours ${ }^{36}$. Jean STAROBINSKI nous apprend que, quand Rousseau met l'accent sur l'historicité de ce qui est artificiel, corrompu chez l'homme, comme quand il parle de la difficulté à démêler l'originaire et le factice, il aura été précédé et guidé par Buffon ${ }^{37}$.

33. Buffon, cité par M. Duchet, art. cité, p. 35.

34. Ibid., p. 36.

35. Michèle DUCHET, art. cité, p. 36.

36. Sur ce point, voir Jean MOREL, "Recherches sur les sources du Discours sur l'origine de l'Inégalité» in Annales de la société JJ. Rousseau, V, 1909, pp. 119-198.

37. Jean StAROBINSKI, J.J. Rousseau, la transparence et l'obstacle, Paris, Gallimard, 1971, p. 382. 
Cependant, la confiance en l'autorité de Buffon, l'admiration portée aux recherches de celui-ci n'ont pas empêché Rousseau de rejeter certaines thèses chères au naturaliste, thèses qui portent précisément sur la nature de l'homme.

On a vu que, pour Buffon, la pensée prouve la spiritualité de l'âme. C'est la pensée - dont le fait de commander aux bêtes, la communication par la parole et le progrès dans les ouvrages sont les effets visibles - qui démontre "l'excellence de notre nature, et la distance immense que la bonté du Créateur a mise entre l'homme et la bête ${ }^{38}$.» Cette preuve n'est nullement convaincante, estime Rousseau. Adoptant la thèse du sensualisme, il ne dénie pas tout entendement à l'animal et admet que, du point de vue de l'entendement, il y a seulement une différence de quantité entre l'homme et l'animal: "Tout animal a des idées puisqu'il a des sens, il combine même ses idées jusqu'à un certain point et l'homme ne diffère de la bête à cet égard que du plus au moins ${ }^{39}$.»

Qu'est-ce qui prouve donc la spiritualité de l'âme?

L'analyse des réactions de l'homme comme de l'animal face aux sollicitations de la nature fournira la preuve décisive. Le comportement de l'animal est tout déterminé : il ne s'écarte pas de la règle qui lui est prescrite, même quand il lui serait avantageux de le faire. Au contraire, l'homme «se reconnaît libre d'acquiescer ou de résister» et s'écarte souvent de la règle à son préjudice. C'est dans cette conscience de la liberté que se montre donc la spiritualité de l'âme humaine ${ }^{40}$.

Déniant à la raison le mérite de distinguer l'homme de l'animal, Rousseau lui dénie aussi le pouvoir de rendre l'homme meilleur. À l'instar de Buffon, il reconnaît que l'homme possède la faculté de se perfectionner. Mais alors que Buffon identifie la perfectibilité - due à la nature d'être pensant de l'homme - à la capacité de progrès à tous égards, Rousseau refuse d'y voir une manifestation de la grandeur, de la moralité, et une condition du bonheur de l'homme:

38. BuFFON, ouv. cité, p. 47.

39. O.C., III, p. 141.

40. Voir O.C., III, p. 142. 
Il serait triste pour nous d'être forcés de convenir que cette faculté distinctive [la perfectibilité], et presque illimitée est la source de tous les malheurs de l'homme ; (...) que c'est elle, qui faisant éclore avec les siècles ses lumières et ses erreurs, ses vices et ses vertus, le rend à la longue le tyran de lui-même et de la Nature. ${ }^{41}$

Face à cette critique, Buffon répondrait sans doute que la société civile avec toutes ses institutions, ouvrage de la raison, constitue à elle seule le plus grand bien qui contrebalance tout mal que peut causer la raison ${ }^{42}$.

Un tel éloge de la raison n'est nullement justifié aux yeux de Rousseau, car un regard «tranquille et désintéressé» porté sur la réalité sociale quotidienne ne lui montre que «la dureté des uns», "l'aveuglement des autres» ${ }^{43}$. Ainsi, par ses ouvrages que sont les institutions sociales, la raison, loin de rendre les hommes meilleurs, n'a en fait que contribué à les rendre méchants et soumis à la servitude. Pour l'auteur de l'Émile, la perfectibilité n'est donc pas cette capacité de progrès indéfini exaltée par Buffon ainsi que par Turgot et Condorcet ${ }^{44}$. Elle est la possibilité de se donner un mode d'existence et une nature autres que ceux que l'homme reçoit de «la Providence». Ce mode d'existence, cette nature peuvent le rendre pleinement humain ou, au contraire, le faire retomber "plus bas que la bête même». Cette dernière possibilité est celle qui s'est réalisée dans la vie sociale existante. Mais s'il en est ainsi, il faudrait remettre en question l'idée d'un état originel où l'homme serait barbare, pervers et dépendant. Il faudrait y voir sans doute une de ces «anciennes erreurs》 à redresser, un de ces "préjugés invétérés à détruire» ${ }^{45}$.

Les doctrines religieuses de Calvin, de Saint Augustin, la philosophie de Hobbes et l'histoire naturelle de Buffon offrent

41. Ibid.

42. «L'homme... n'est fort, il n'est grand, il ne commande à l'univers que parce qu'il a su se commander à lui-même, se dompter, se soumettre, et s'imposer des lois; l'homme en un mot n'est homme que parce qu'il a su se réunir à l'homme."; "Cette réunion est de l'homme l'ouvrage le meilleur, c'est de sa raison l'usage le plus sage." (BuFfoN, cité par M. DUCHET, art. cité, p. 11-12).

43. O.C., III, p. 126-127.

44. Sur ces auteurs, voir G. GUSDORF, ouv. cité, p. 319; 332.

45. O.C., III, p. 160 . 
des images de l'homme de différents points de vue et, de ce fait, ne présentent pas les mêmes thèses, ni ne mettent en œuvre les mêmes arguments. Pourtant, à chaque fois, la critique de Rousseau tend vers l'affirmation de l'homme comme être naturellement bon et libre.

La bonté naturelle et la liberté originelle de l'homme sont des évidences aux yeux de Rousseau. C'est pour ne les avoir pas retrouvées dans les conceptions anthropologiques de l'époque qu'il déplore le retard de la connaissance de l'homme par rapport aux autres branches du savoir.

L'ignorance de ces deux principes est, au jugement de Rousseau, à l'origine de l'état «d'incertitude et d'obscurité sur la véritable définition du droit naturel» ${ }^{46}$. C'est encore cette ignorance qu'on décèle dans le vice de méthode des "politiques» et des «philosophes» : partir de l'homme dans sa réalité actuelle pour retrouver la nature. Cette erreur n'est nulle part plus flagrante que dans la conception de ces auteurs, qui, de la patience dont font preuve les esclaves, infèrent l'existence d'un " penchant naturel à la servitude». S'ils étaient partis de la nature de l'homme, c'est-à-dire de la nature d'un être libre, ils auraient vu que la servitude représente un asservissement et non l'objet des «dispositions naturelles de l'homme» ${ }^{47}$. C'est aussi la même ignorance de la bonté et de la liberté originelles qui fait croire que l'inégalité socio-politique a un fondement naturel.

Mais les intuitions de Rousseau - déjà présentées dans le Discours sur les sciences et les arts ${ }^{48}$ - ont besoin d'être développées et démontrées, d'autant plus qu'elles ne s'accordent nullement avec l'expérience, qui impose plutôt l'image d'une condition humaine liée à la méchanceté et à la servitude ${ }^{49}$.

Ce sera la tâche du Discours sur l'Inégalité d'entreprendre cette démonstration.

Département de philosophie

Université de Moncton et d'Ottawa

46. $O . \dot{C} .$, III, p. 124.

47. O.C., III, p. 181.

48. Voir O.C., III, pp. $7 ; 22$.

49. "Les hommes sont méchants; une triste et continuelle expérience dispense de la preuve» (O.C., III, p. 202); "L'homme est né libre, et partout il est dans les fers» (Ibid., p. 351). 